\title{
Tos crónica como presentación de una enfermedad infrecuente
}

\author{
PAULA BARCOS M.*., CRISTÓBAL RAMOS G.**, JUAN CARLOS DÍAZ P.**, \\ ELY JOVER L.* CRISTINA FERNÁNDEZ F.*** y MAURICIO ALAMO T.****
}

\section{Chronic cough as presentation of a rare disease}

Se presenta un hombre de 42 años con diagnóstico de resistencia a Insulina en tratamiento con metformina $850 \mathrm{mg} /$ día, atorvastatina 10 $\mathrm{mg}$ /día. Trabaja en empresa de metalmecánica (jefe), expuesto ocasionalmente a polvo metálico. Septoplastía nasal en enero de 2012. Refiere que tras un resfrío común (marzo de 2012) permaneció con tos que no cedió por lo que consultó en varias ocasiones. Cuatro meses después fue hospitalizado con diagnóstico de obstrucción bronquial difusa (tos, disnea, sibilancias y dolor esternal). En esa oportunidad no se encontró agente infeccioso (panel viral, serología Mycoplasma, Bordetella, antígeno urinario Neumococo y Legionella todos negativos) y la tomografía computarizada (TAC) de tórax fue informada como prácticamente normal (salvo por atelectasia lineal en LID). Cuantificación de inmunoglobulinas, electroforesis de proteínas, perfil hepático, $\mathrm{TSH}, \operatorname{IgE}, \beta 2$ microglobulina, todos en rangos normales. ANA negativo. Hemograma de la hospitalización: hemoglobina 10,4 g/dL, hematocrito $32 \%$ y VHS $100 \mathrm{~mm} / \mathrm{h}$. Ferritina 821,1 UI/L. Nasofaringolaringoscopía: sugerente de reflujo faringolaríngeo. VIH negativo. ECG: taquicardia sinusal sin signos de isquemia.

Fue dado de alta tras estabilización de sín- tomas, con $20 \mathrm{mg}$ de prednisona diarios que mantuvo por 4 meses hasta consultar nuevamente. En esa oportunidad destacaba al examen físico: disfonía, tos persistente que interrumpía la conversación, rinolalia discreta, murmullo pulmonar disminuido leve global, espiración prolongada y sibilancias escasas difusas. Se planteó Asma bronquial como hipótesis diagnóstica e inició tratamiento con Salmeterol/Fluticasona (100 $\mu \mathrm{g} / 1.000 \mu \mathrm{g}$ diarios), continuó con prednisona, omeprazol, Fluticasona nasal y Loratadina en espera de exámenes.

La espirometría evidenció una alteración ventilatoria obstructiva moderada que no se modificó con salbutamol (valores basales: $\mathrm{VEF}_{1}$ : 1,62 L (48\%), CVF: 2,66 L (65\%) $\mathrm{VEF}_{1} / \mathrm{CVF}: 0,61$; con salbutamol: $\mathrm{VEF}_{1}$ : $1,73 \mathrm{~L}$ y CVF: $2,71 \mathrm{~L}$ ). La tos disminuyó en un $80 \%$ tras dos semanas de iniciar este esquema terapéutico. Sin embargo, el paciente seguía con dolor retroesternal que se reproducía a la palpación. El hemograma de control reveló hemoglobina de 13,4 g/dL, hematocrito $34 \%$, VHS $14 \mathrm{~mm} / \mathrm{h}$. Reapareció tos y dolor tras disminuir dosis de prednisona a $10 \mathrm{mg} /$ día y se solicitó TAC de tórax (Figura 1), dado que el paciente continuaba con dolor y tos a pesar de tratamiento bien llevado.

\footnotetext{
* Neumólogo, Hospital Clínico Universidad de Chile.

** Radiólogo, Hospital Clínico Universidad de Chile.

*** Patóloga, Hospital Clínico Universidad de Chile.

**** Reumatólogo, Clínica Dávila.
} 


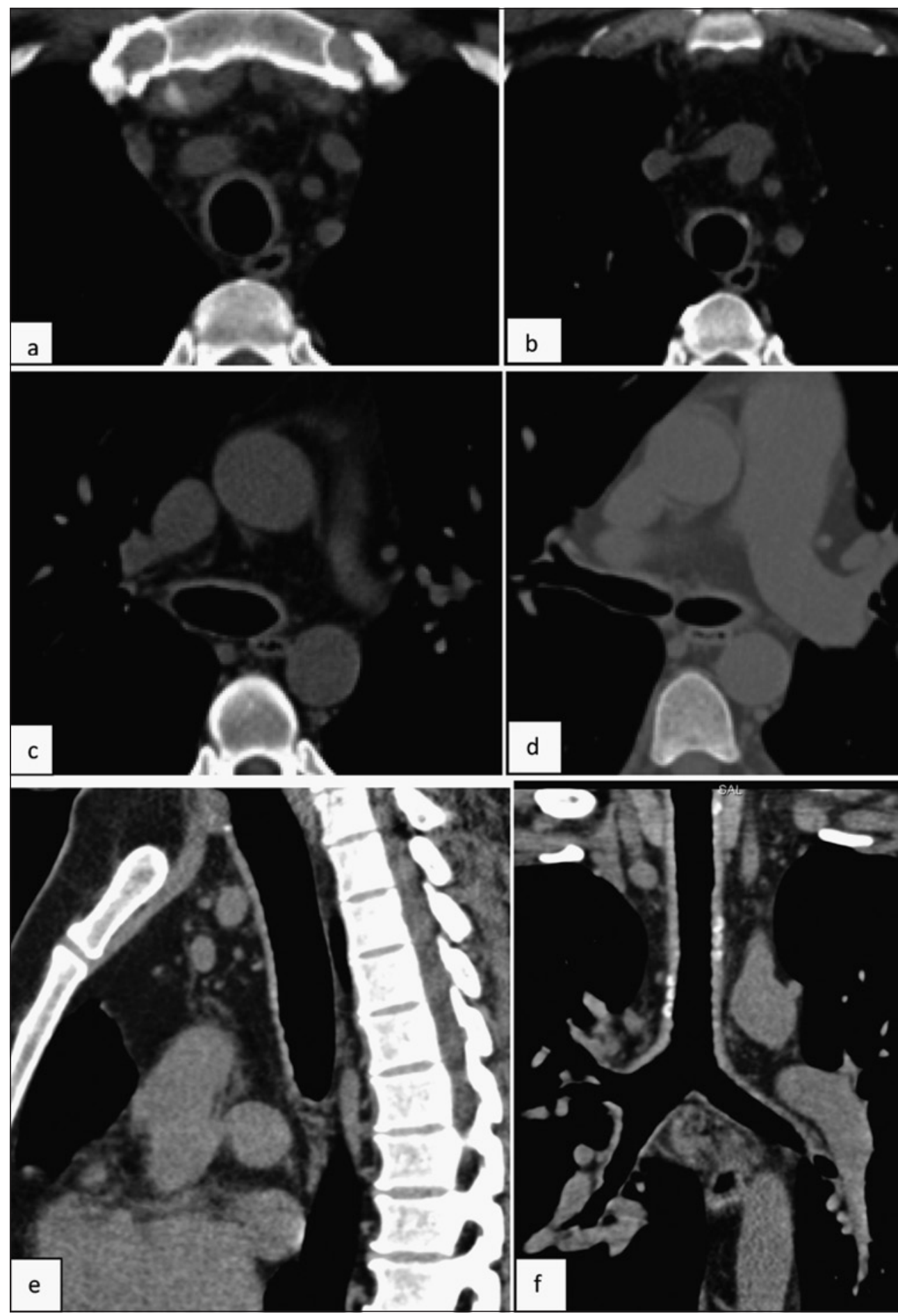

Figura 1. Los cortes en plano axial (a-d) muestran aumento de la atenuación de las paredes traquales, y engrosamiento liso de la pared anterior y lateral de ésta con respeto de la pared posterior membranosa. Se aprecia además una calcificación focal en el espesor de la pared traqueal y aumento de grosor concéntrico de la pared de los bronquios fuentes y lobares. El corte sagital (e) muestra la diferencia de grosor de la pared anterior vs la pared posterior membranosa respetada. En el corte coronal (f) se observa el extenso compromiso de la vía aérea comprometiendo globalmente la tráquea y la extensión hacia bronquios fuentes y lobares. 


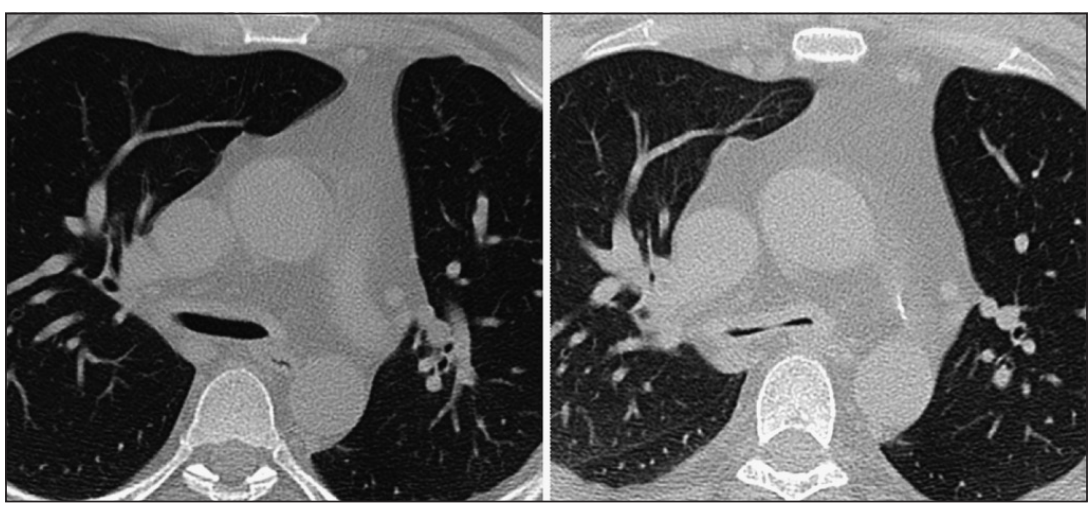

Figura 2. En el lado izquierdo se observa el corte axial a nivel de la carina en inspiración, con el engrosamiento de las paredes bronquiales. En el cuadro de la derecha, al mismo nivel, pero en espiración forzada, se aprecia el colapso de más de un $50 \%$ del lumen de la vía aérea.

\section{¿Cual es su diagnóstico?}

En base a los hallazgos de la TAC de tórax se hizo el diagnóstico clínico radiológico de Policondritis recidivante. El paciente continuó con prednisona, en dosis de $40 \mathrm{mg} /$ día. ANA, ANCA, AntiDNA, ENA, FR, todos negativos. El paciente no tiene manifestaciones en pabellones auriculares ni en la nariz. No hay evidencias de compromiso cardíaco hasta el momento.

Fue evaluado en conjunto con reumatólogo y se decidió inciar ciclofosfamida en pulsos y luego azatioprina $150 \mathrm{mg}$ /día v.o. con respuesta clínica favorable: ha disminuido la tos y la disnea en $80 \%$ y el paciente ha mejorado su calidad de vida.

\section{Discusión}

La policondritis recidivante ${ }^{1,2}$ es una entidad considerada rara en la literatura internacional. Es una enfermedad multisistémica, crónica, progresiva, debilitante y discapacitante. Se caracteriza por episodios recurrentes de inflamación de las estructuras cartilaginosas involucradas, principalmente oídos, nariz, tráquea y bronquios principales. La edad más frecuente de presentación es en la década de los 40 y su distribución es equitativa entre hombres y mujeres.

El compromiso de las vías respiratorias ${ }^{3}$ ocurre en aproximadamente la mitad de los pacientes durante su evolución y no es la forma de presentación inicial más frecuente. Habitualmente los pacientes desarrollan manifestaciones auriculares y nasales antes que las respiratorias.

El compromiso de vías aéreas se considera un signo de mal pronóstico dado que al ser una enfermedad progresiva, evoluciona con cambios fibróticos y estrechamiento difuso de ésta con alteraciones en el flujo de aire ${ }^{4}$. De hecho, más del $50 \%$ de las muertes en pacientes con esta enfermedad se atribuyen al compromiso respiratorio. Por otro lado, se produce inflamación cartilaginosa persistente y alteración de la función mucociliar. Estos factores más el uso de inmunosupresores como tratamiento de la inflamación crónica, predisponen al paciente a infecciones respiratorias recurrentes.

También se ha descrito el compromiso de cartílagos costoesternales (muy raro) como muy probablemente presentó este paciente. Este hecho dificulta aún más la respiración y la calidad de vida de quienes tienen Policondritis Recidivante.

La dificultad diagnóstica de los pacientes que inician esta enfermedad con compromiso respiratorio, es que la mayoría de las veces el diagnóstico se retarda dado que las manifestaciones iniciales habitualmente son tos y disnea como en el paciente que presentamos. Es común que estos síntomas inespecíficos sean interpretados como asma o limitación crónica al flujo aéreo y que reciban esteroides inhalados o sistémicos con respuesta clínica parcial. Además, las pruebas de función pulmonar son poco específicas ${ }^{5}$. Sólo cuando el clínico considera que el paciente continúa sintomático a pesar de tratamiento es que se plantea investigar otras patologías mediante imágenes como en este caso. Cuando aparece compromiso respiratorio en pacientes con policondritis, el diagnóstico es obvio. En el examen físico pulmonar no hay signos que lo sugieran. De los hallazgos más frecuentes cuando estos existen, es el estridor.

Característicamente la TAC de tórax puede mostrar lesiones laringotraqueales y anormalidades en la tráquea y grandes bronquios. Las manifestaciones más comunes son el aumento de atenuación y engrosamiento liso de las paredes de la vía aérea, con o sin calcificaciones, siendo menos frecuentes la estenosis traqueal $\mathrm{y} / \mathrm{o}$ 
bronquial ${ }^{6}$. El engrosamiento de la pared anterior y lateral con respeto de la pared posterior membranosa es prácticamente patognomónica de esta enfermedad (Figura 1). Además, el compromiso del cartílago predispone al colapso de la vía aérea en espiración (traqueomalacia), lo que es mejor evaluado con TAC de tórax dinámica en espiración forzada ${ }^{7}$ (Figura 2). La obstrucción del flujo aéreo puede conducir a atrapamiento aéreo y oligohemia pulmonar, atelectasias, neumonitis obstructiva o bronquiectasias ${ }^{8}$. En un estudio de 18 pacientes con esta patología, la TAC dinámica en espiración mostró atrapamiento aéreo lobulillar en 4 de ellos, en ausencia de compromiso de la vía aérea principal, lo que sugiere que la vía aérea pequeña también puede ser comprometida ${ }^{9}$. La resonancia magnética podría tener un papel en el control de estos pacientes determinando actividad de la enfermedad a través de demostración de aumento de la señal de la pared en secuencias potenciadas en T2 y aumento de la señal en secuencias potenciadas en $\mathrm{T} 1$ posterior al uso de Gadolinio como medio de contraste ${ }^{10}$.

La broncoscopía es el paso diagnóstico siguiente en pacientes con tos y disnea que no responde a tratamiento ${ }^{11}$, pero puede desencadenar inflamación traqueal y bronquial por lo que no se aconseja.

Los diagnósticos diferenciales de la estrechez traqueal difusa incluyen procesos inflamatorios, neoplásicos e infecciosos. En los adultos, la tuberculosis traqueal es rara y se acompaña de lesiones cavitadas en el parénquima. Otros diagnósticos diferenciales son la amiloidosis y la sarcoidosis cuyos hallazgos radiológicos permiten hacer la diferencia.

Los hallazgos histopatológicos de los pacientes que se someten a biopsia traqueal corresponden a pérdida de la tinción basofílica de la matriz cartilaginosa, infiltración pericondral de células redondas y destrucción del cartílago con reemplazo fibroso. Las biopsias endobronquiales no proveen de cartílago por lo que no son útiles en el diagnóstico.

El tratamiento se basa en inmunosupresión con esteroides solos o en combinación con otro inmunosupresor, a pesar de que no existen trabajos randomizados y prospectivos que lo avalen dada su condición de enfermedad rara. Tampoco se ha establecido la duración óptima del tratamiento ni las dosis más efectivas. La recomendación de expertos es iniciar el tratamiento con Prednisona $1 \mathrm{mg} / \mathrm{kg} /$ día por 8 a 12 semanas y reevaluar al paciente en forma periódica para mantener al paciente con la mínima dosis que permita su estabilidad clínica. Se ha utilizado azatioprina, ciclofosfamida y otros como metotrexato y antiTNF $\alpha$.

La evolución de la enfermedad es variable, describiéndose casos de estabilidad con terapia inmunosupresora, remisión espontánea, progresión hacia estenosis crítica de la tráquea y muerte a pesar de intentos de manejo de la vía aérea con stents endobronquiales.

\section{Bibliografía}

1.- MCADAM L P, O'HANLAN M A, BLUESTONE R, PEARSON C M. Relapsing polychondritis: prospective study of 23 patients and a review of the literature. Medicine (Baltimore) 1976; 55: 193-215.

2.- LETKO E, ZAFIRAKIS P, BALTATZIS S, VOUDOURI A, LIVIR-RALLATOS C, FOSTER C S. Relapsing polychondritis: a clinical review. Semin Arthritis Rheum 2002; 31: 384-95.

3.- ENG J, SABANATHAN S. Airway complications in relapsing polychondritis. Ann Thorac Surg 1991; 51: 686-92.

4.- LEE K S, ERNST A, TRENTHAM D E, FELLER-KOPMAN D J, BOISELLE P M. Relapsing polychondritis: prevalence of expiratory CT airway abnormalities. Radiology 2006; 240: 565-73.

5.- MOHSENIFAR Z, TASHKIN D P, CARSON S A, BELLAMY P E. Pulmonary function in patients with relapsing polychondritis. Chest 1982; 81: 711-7.

6.- KILMAN W J. Narrowing of the airway in relapsing polychondritis. Radiology 1978; 126: 373-6.

7.- TILLIE-LEBLOND I, WALLAERT B, LEBLOND D, SALEZ F, PÉREZ T, REMY-JARDIN M, et al. Respiratory involvement in relapsing polychondritis. Clinical, functional, endoscopic, and radiographic evaluations. Medicine (Baltimore) 1998; 77: 168-76.

8.- BEHAR J V, CHOI Y W, HARTMAN T A, ALLEN N $\mathrm{B}$, MCADAMS H P. Relapsing polychondritis affecting the lower respiratory tract. AJR Am J Roentgenol 2002; 178: 173-7.

9.- DAVIS S D, BERKMEN Y M, KING T. Peripheral bronchial involvement in relapsing polychondritis: demonstration by thin-section CT. AJR Am J Roentgenol 1989; 153: 953-4.

10.- HEMAN-ACKAH Y D, REMLEY K B, GODING JR $\mathrm{G} \mathrm{S}$. A new role for magnetic resonance imaging in the diagnosis of laryngeal relapsing polychondritis. Head Neck 1999; 21:484-9.

11.- MAIMON N, MARRAS T, HWANG D, PAUL N, KESHAVJEE S, CHAN C K N. A 46-year-old woman with dyspnea, stridor and chronic cough. Eur Respir J 2006; 28: 666-9.

Correspondencia a:

Dr. Juan Carlos Díaz P.

Depto. Radiología

Hospital Clínico Universidad de Chile.

E-mail: diazpatino@vtr.net 